\title{
Comparing models for the ground state energy of a trapped one-dimensional Fermi gas with a single impurity
}

\author{
N. J. S. Loft, L. B. Kristensen, A. E. Thomsen, and N. T. Zinner \\ Department of Physics and Astronomy, Aarhus University, DK-8000 Aarhus C, Denmark
}

(Dated: September 11, 2018)

\begin{abstract}
We discuss the local density approximation approach to calculating the ground state energy of a one-dimensional Fermi gas containing a single impurity, and compare the results with exact numerical values that we have for up to 11 particles for general interaction strengths and up to 30 particles in the strongly interacting case. We also calculate the contact coefficient in the strongly interacting regime. The different theoretical predictions are compared to recent experimental results with few-atom systems. Firstly, we find that the local density approximation suffers from great ambiguity in the few-atom regime, yet it works surprisingly well for some models. Secondly, we find that the strong interaction theories quickly break down when the number of particles increase or the interaction strength decreases.

PACS numbers: 67.85.-d,03.65.Ge,05.30.Fk
\end{abstract}

\section{INTRODUCTION}

As cold atomic gas capabilities advance [1, 2], a large number of interesting quantum systems can now be built using the cold atom toolbox [3 5]. The structure and dynamics of one-dimensional quantum systems is a specific venue in which cold atoms have driven a new surge of excitement as a number of important models of manybody quantum physics can now be probed experimentally [6 14]. This includes the famous strongly interacting Bose systems studied by Tonks [15] and Girardeau [16]. More recently, it has become possible to push these studies to smaller particle numbers [17] so that few-body physics in one-dimensional traps with controllable interactions can now be realized. A number of such studies in recent years using two-component Fermi systems have probed such concepts as fermionization [18], pairing [19], polarons 20], and the Hubbard 21] and Heisenberg models [22], all in the limit where the system contains just a few particles. These developments have sparked a lot of interest in the few-body community and a number of new theoretical studies looking at various aspects of these twocomponent Fermi systems in one dimension have emerged 23 49].

In the present paper we are interested in the recent experiments that have shown how one may build a Fermi system consisting of an impurity and a Fermi sea one particle at a time 20]. This amounts to building a so-called Fermi polaron from a few-body perspective. The experimental results presented in Ref. 20] suggest that already for a system consisting of only six particles (five identical fermions and a single impurity of the same mass as the other particles) one can see the emergence of a manybody system taking place. This is a rather remarkable result and in the present paper we would like to explore this in more detail. The experimental results consists of a series of measurements of the energy for different interaction strengths and for different system sizes and have previously been compared to theoretical predictions in the literature [30]. However, there are some ambigu- ities in this comparison arising from the fact that the experimental system is in a trap while a lot of theoretical work considers a homogeneous system. Proposals for merging the homogeneous results with a trapped system are known and one of our goals here is to assess how well they work as a function of particle number and interaction strength. We will take advantage of the fact that we have access to both very accurate numerical calculations for all interaction strengths for up to eleven particles and in the strongly interacting regime for up to thirty particles in a harmonic trap. Using these results we will shed some light on the questions concerning how many particles constitutes 'many' in one-dimensional impurity systems and when such systems can be considered in the strongly interacting regime.

\section{MODEL}

We consider a system of $N$ spin- $\frac{1}{2}$ fermions with mass $m$ confined in a one-dimensional (1D) harmonic trap with oscillator frequency $\omega$. The fermions in the gas are spinpolarized with $N-1$ particles in, say, the spin-up state, while the remaining particle (the impurity) is in the spindown state. The particles interact via a short-range interaction modelled by a delta function of coupling strength $g$. The system is described by the Hamiltonian

$$
H=\sum_{i=1}^{N}\left[-\frac{\hbar^{2}}{2 m} \frac{\partial^{2}}{\partial x_{i}^{2}}+\frac{1}{2} m \omega^{2} x_{i}^{2}\right]+g \sum_{i<j} \delta\left(x_{i}-x_{j}\right) .
$$

In the interaction term, the sum runs over all pairs of particles, but due to the antisymmetry of the total wave function under exchange of any two majority particles, the majority particles do not couple to each other. Thus the interaction term above is equivalent to only summing over the $N-1$ pairs consisting of the impurity and each of the majority particles. As has been recently demonstrated experimentally, the interaction strength may be varied from small to large positive and negative values 
[18]. We will limit ourselves to a repulsive interaction, $g>0$. Throughout this paper, we use harmonic oscillator units where $\omega=m=\hbar=1$.

\section{STRONG COUPLING REGIME}

We start by discussing the Tonks-Girardeau (TG) limit in which the interaction between the particles is infinitely strong, $1 / g \rightarrow 0$. In this limit, the ground state becomes $N ! /((N-1) ! \cdot 1 !)=N$-fold degenerate with the energy $E_{\infty}^{\mathrm{t}}=N^{2} / 2$, and the wave function vanish whenever any two particles meet. The superscript $t$ denotes the fact that the system is trapped, and the subscript $\infty$ refers to the interaction being infinitely strong. Notice that the degenerate energy in the ground state manifold is also the ground state energy of an ideal non-interacting Fermi gas of $N$ particles in a harmonic trap, i.e. the particles occupy the $N$ lowest-lying energy states in the trap.

The degeneracy is lifted by moving slightly away from $1 / g=0$, and the wave function is lifted slightly away from zero at the surfaces where the impurity meets a majority particle. This allows the impurity to switch position with its neighbors, and we can therefore think of the particles as occupying sites on a lattice. The system can be described by an effective Heisenberg spin chain model as discussed in [36, 37, 44, 47]. To linear order in $1 / g \ll 1$, we may cast the Hamiltonian as

$$
H=E_{\infty}^{\mathrm{t}}-\frac{1}{g} \sum_{k=1}^{N-1} \frac{\alpha_{k}}{2}\left[1-\boldsymbol{\sigma}^{k} \cdot \boldsymbol{\sigma}^{k+1}\right],
$$

where $\boldsymbol{\sigma}^{k}=\left(\sigma_{x}^{k}, \sigma_{y}^{k}, \sigma_{z}^{k}\right)$ are the Pauli matrices acting on the spin of the particle at site $k$, and $\alpha_{k}$ is a geometric coefficient determined by the trap potential. These are the same $\alpha$-coefficients discussed in [44], where the case of a general confining potential, $V(x)$, and an arbitrary number of spin-up and spin-down particles is considered. The general case captures the behavior of both bosonic and fermionic particles near the TG limit. In Ref. [56] it is shown that the geometric coefficient $\alpha_{k}$ generally can be expressed as:

$$
\begin{aligned}
\alpha_{k}= & 2 \sum_{i=1}^{N} \sum_{j=1}^{N} \sum_{l=0}^{N-1-k} \frac{(-1)^{i+j+N-k}}{l !}\left(\begin{array}{c}
N-l-2 \\
k-1
\end{array}\right) \\
& \times \int_{a}^{b} \mathrm{~d} x \frac{2 m}{\hbar^{2}}\left(V(x)-E_{i}\right) \psi_{i}(x) \frac{\mathrm{d} \psi_{j}}{\mathrm{~d} x} \\
& \times\left[\frac{\partial^{l}}{\partial \lambda^{l}} \operatorname{det}\left[(B(x)-\lambda \mathbf{I})^{(i j)}\right]\right]_{\lambda=0} \\
& +\sum_{i=1}^{N}\left[\frac{\mathrm{d} \psi_{i}}{\mathrm{~d} x}\right]_{x=b}^{2} .
\end{aligned}
$$

To compute this we need access to the $N$ lowestenergy single-particle wavefunctions $\psi_{i}(x)$ solving the Schrödinger equation $\left[\frac{-\hbar^{2}}{2 m} \frac{\partial^{2}}{\partial x^{2}}+V(x)\right] \psi_{i}=E_{i} \psi_{i}$. In the present case, this is the analytically solved Schrödinger equation for a single particle in a harmonic oscillator. In Eq. (3) $B(x)$ is a $N \times N$ symmetric matrix with the $m n$ 'th entry given by $\int_{a}^{x} \mathrm{~d} y \psi_{m}(y) \psi_{n}(y)$, and ()$^{(i j)}$ defines a minor obtained by removing the $i$ 'th column and the $j$ 'th row. For most potentials such as smooth potentials like the present case, the integration limits will be $b=\infty=-a$, and for some non-analytic potentials such as a hard box, the integration will be confined to some finite region of space. For symmetric confining potentials, as the one studied in this paper, the coefficients are also symmetric, $\alpha_{k}=\alpha_{N-k}$.

The main motivation for the present work is that we are able to determine these geometric $\alpha$-coefficients exactly in the general case of an arbitrary potential, see Ref. 56 for a detailed presentation of our method including a derivation of Eq. (3). The method is implemented as a highly efficient numerical program released as open source software [59]. We stress the importance of our capability to perform these calculations numerically exact; we do not use Monte Carlo integration or any other approximate method. For this paper, we calculate the geometric coefficients numerically exact for a harmonic potential for $N \leq 30$. The coefficients are given in Section A.Calculating the coefficients for such a high number of particles as 30 is an extremely demanding computational task. However, the method we have developed enables us to perform these calculations on a small laptop to a very high level of precision in a matter of hours for 30 particles. For a few particles, the calculations are done almost immediately.

Since the harmonic potential is such a well-studied confining potential, this allows us to compare our exact results with the approximate methods studied in the literature. In particular, we are interested in comparing methods for calculating the ground state energy in the vicinity of the TG limit. To linear order in $1 / g$, we write the ground state energy as

$$
E_{g}^{\mathrm{t}}=E_{\infty}^{\mathrm{t}}-\frac{1}{g} \mathcal{C}
$$

where $\mathcal{C}$ is the eigenvalue of the sum in Eq. (2) for the ground state, i.e. the largest eigenvalue. We denote $\mathcal{C}$ the contact coefficient [50, 51]. Access to the exact geometric coefficients $\alpha_{i}$ allows us to diagonalize the Hamiltonian in Eq. (2) and calculate the contact coefficient exactly. For $N \leq 30$ we compute $\mathcal{C}$ and want to compare our results with previous approximate formulas for this constant.

\section{A. Previous results}

Now we will review some previous calculations of the contact coefficient $\mathcal{C}$ in Eq. (44). However, since the previous theories may not take the trap into account, we will have to adapt the existing formulas in order to directly compare them with our results. In the following, 
we will distinguish between the free system and the (harmonically) trapped system. Furthermore, we will discuss different regimes of the interaction strength $g$ and the particle number $N$.

A pioneering piece of work was done by McGuire [52] who calculated the ground state energy for a free 1D Fermi gas with a single impurity for an arbitrary interaction strength $g$ subject to periodic boundary conditions. Thus the trap needs to be taken into account. This problem was recently addressed by Astrakharchik and Brouzos who applied the local density approximation (LDA) to McGuire's result in order to obtain an expression for a harmonically trapped system [30]. However, McGuire assumes that the number of particles $N$ is even and large. This subtlety does not appear to be explicitly discussed in Ref. 30]. Namely, the implicit condition that $N$ is very large makes it interesting to compare the result with our exact results for small $N$. To get the desired contact coefficient $\mathcal{C}$, we should expand Astrakharchik and Brouzos' LDA result around $1 / g=0$ to first order and read off the coefficient.

McGuire's energy expression was found for a free interacting system subject to periodic boundary conditions (PBC). It would be interesting to consider a hard wall boundary condition (HWC), too. This problem was solved by Oelkers et al. for a free gas in the strong coupling regime [53] which is exactly the regime we are interested in. In fact Oelkers et al. found results for both PBC and HWC for finite (even) $N$, and not just in the limit of large $N$. Just as Astrakharchik and Brouzos applied LDA to McGuire's free result, we shall apply LDA to Oelkers' free results. Since the LDA method maps the free system to a trapped system, we would intuitively think that HWC would produce a better final result, since a hard wall would resemble a harmonic potential more that a periodic boundary. This will turn out not to be the case, but as we shall also see, these LDA results are subject to high uncertainties in the small $N$ regime. Nevertheless this is an important regime as current experiments study the behaviour of few particles.

The starting point for our analysis are the results summarized in Table I. Here we have recorded whether the energy is for a harmonically trapped or a free system, which boundary condition is used and which values of the interaction strength and particle numbers it is valid for.

\begin{tabular}{lcccc} 
Reference & Trapped & BC & $g$ & $N$ \\
\hline McGuire [52] & No & PBC & any & $N \rightarrow \infty$ \\
Astra. [30] & Yes & PBC & any & $N \rightarrow \infty$ \\
Oelkers [53] & No & PBC & $g \rightarrow \infty$ & even \\
Oelkers [53] & No & HWC & $g \rightarrow \infty$ & even
\end{tabular}

TABLE I: Summary of some previous results.

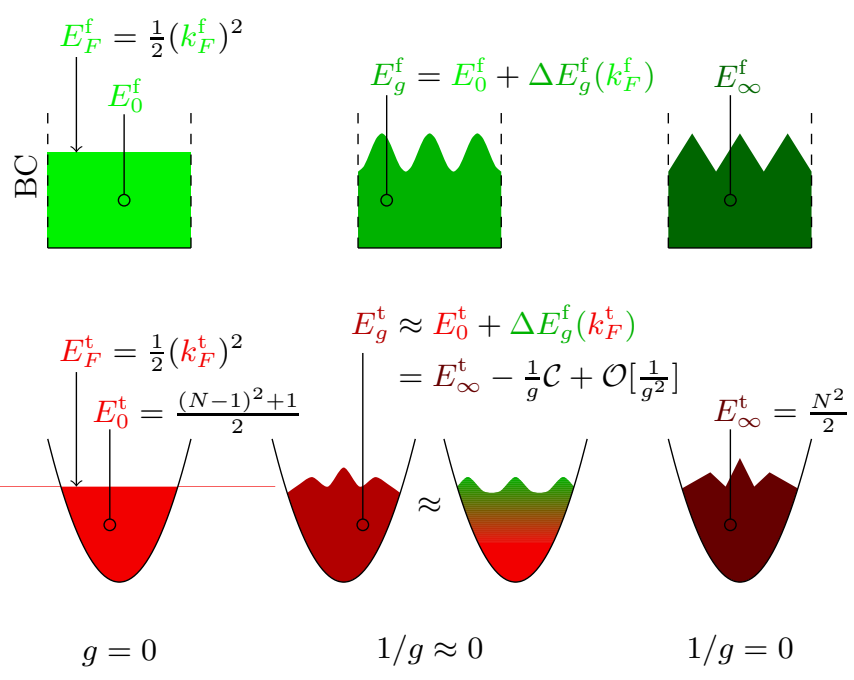

FIG. 1: Illustration of the local density approximation. The top part of the picture shows the free system solved for some boundary condition (green), whereas the bottom part shows the trapped system (red). As a way of representing an interacting sea of particles, we have drawn waves on the surface with increasing sharpness for increasing interaction strength. This should only be understood as an intuitive guide to the eye. The Fermi level is defined in the non-interacting case and denoted by an arrow, while the total energy of the system is denoted by a circle.

\section{B. The local density approximation}

The strategy to map a result from the free case to the trapped case is by applying LDA. Therefore, we will briefly discuss LDA, and most importantly we will encounter a weakness in the method for small values of $N$.

Denote by $E_{0}^{\mathrm{f}}\left(E_{0}^{\mathrm{t}}\right)$ the energy of the free (trapped), non-interacting system. Suppose that we know the energy states of these two systems, and that we therefore can associate with them Fermi levels and Fermi momenta, denoted $E_{F}^{\mathrm{f}}\left(E_{F}^{\mathrm{t}}\right)$ and $k_{F}^{\mathrm{f}}\left(k_{F}^{\mathrm{t}}\right)$, respectively. Suppose furthermore that we know an expression for the energy of the interacting system, but only in the free case. Denote this energy by

$$
E_{g}^{\mathrm{f}}=E_{0}^{\mathrm{f}}+\Delta E_{g}^{\mathrm{f}}\left(k_{F}^{\mathrm{f}}\right),
$$

where $\Delta E_{g}^{\mathrm{f}}$ is the correction due to interaction, which will generally depend on $k_{F}^{\mathrm{f}}$. Now, the quantity sought after is the energy for the interacting and trapped system. Within LDA, this is found by mapping

$$
E_{0}^{\mathrm{f}} \mapsto E_{0}^{\mathrm{t}} \quad \text { and } \quad k_{F}^{\mathrm{f}} \mapsto k_{F}^{\mathrm{t}} .
$$

The interaction correction in the trapped case retain its functional form from the free case, but is now evaluated at the trapped Fermi momentum instead of the free Fermi 


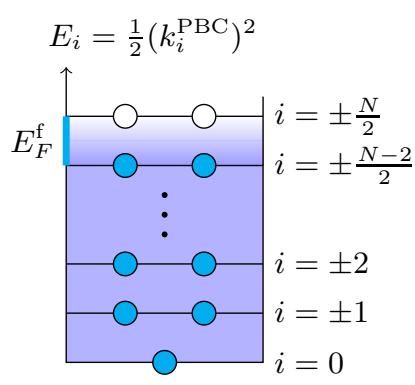

PBC, $N$ even

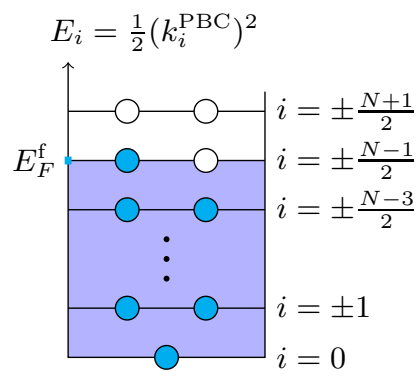

PBC, $N$ odd

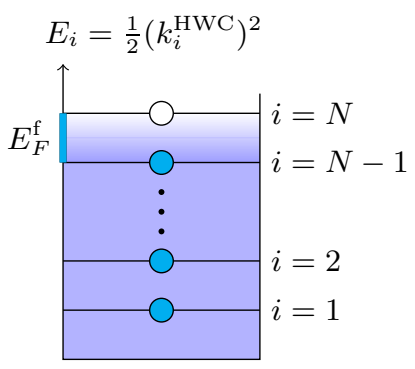

HWC

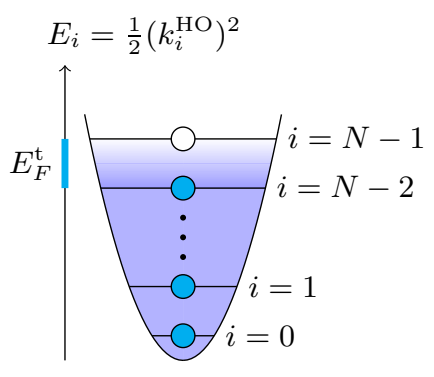

$\mathrm{HO}$

FIG. 2: The Fermi level, $E_{F}^{\mathrm{f}, \mathrm{t}}$, should lie between the highest energy of the occupied states and the lowest energy of the unoccupied states for the non-interacting system. In this limit the $N-1$ states with lowest energy are filled up. Notice that in PBC for $N$ odd, the highest occupied state and the lowest unoccupied state share the same energy, and thus the Fermi level is well-defined, but that we have a certain freedom in the other cases. This freedoms leads us to define the tuning parameters $\lambda^{\mathrm{PBC}}, \lambda^{\mathrm{HWC}}, \lambda^{\mathrm{HO}} \in[0 ; 1]$.

momentum. Thus the LDA expression for the interacting and trapped system is

$$
E_{g}^{\mathrm{t}} \approx E_{0}^{\mathrm{t}}+\Delta E_{g}^{\mathrm{f}}\left(k_{F}^{\mathrm{t}}\right) .
$$

An illustrative sketch of the LDA procedure is shown on Figure 1.

We have just described the LDA method if our starting point was the known non-interacting limit for both the free and trapped system. If, however, we approached a finite interaction starting from the infinitely strong interacting case, $1 / g=0$, we would instead have mapped

$$
E_{\infty}^{\mathrm{f}} \mapsto E_{\infty}^{\mathrm{t}} \quad \text { and } \quad k_{F}^{\mathrm{f}} \mapsto k_{F}^{\mathrm{t}},
$$

where $E_{\infty}^{\mathrm{f}}\left(E_{\infty}^{\mathrm{t}}\right)$ is the energy of the free (trapped) infinitely strongly interacting system, both assumed to be known. We use this approach in Subsection IID.

The employment of LDA relies on the knowledge of the Fermi momenta, $k_{F}^{\mathrm{f}, \mathrm{t}}$, for the free and trapped (noninteracting) systems, or equivalently the Fermi levels, $E_{F}^{\mathrm{f}, \mathrm{t}}$. Now the problem is that the Fermi level (also called the chemical potential) is not well-defined if the systems contains a finite number of particles. We know that the Fermi level should lie between the highest energy of the occupied states and the lowest energy of the unoccupied states. But exactly where between these two energies is irrelevant for the occupancy of the states. The problem is illustrated on Figure 2 for the four relevant situations. In the non-interacting limit, the $N-1$ identical fermions fill up the $N-1$ states of lowest energy. 60. Recall that the momentum for a particle subject to $\mathrm{PBC}, \mathrm{HWC}$ or in a harmonic oscillator is given as

$$
\begin{array}{ll}
k_{i}^{\mathrm{PBC}}=\frac{2 \pi}{L} i, & i=0, \pm 1, \pm 2, \ldots \\
k_{i}^{\mathrm{HWC}}=\frac{\pi}{L} i, & i=1,2,3, \ldots \\
k_{i}^{\mathrm{HO}}=b^{-1} \sqrt{2 i+1}, & i=0,1,2, \ldots
\end{array}
$$

where $L$ is the length of the system and $b=\sqrt{\hbar / m \omega}$ is the standard oscillator length, $b=1$ in our units. Then the energy at the Fermi levels can be described using the following expressions for the Fermi momenta:

$$
\begin{aligned}
& k_{F}^{\mathrm{PBC}}=\left\{\begin{array}{ll}
\frac{\pi}{L}\left(N-2+2 \lambda^{\mathrm{PBC}}\right) & \text { if } N \text { is even } \\
\frac{\pi}{L}(N-1) & \text { if } N \text { is odd }
\end{array},\right. \\
& k_{F}^{\mathrm{HWC}}=\frac{\pi}{L}\left(N-1+\lambda^{\mathrm{HWC}}\right), \\
& k_{F}^{\mathrm{HO}}=b^{-1} \sqrt{2\left(N-\frac{3}{2}+\lambda^{\mathrm{HO}}\right)} .
\end{aligned}
$$

Here $\lambda^{\alpha} \in[0,1]$ with $\alpha=\mathrm{PBC}, \mathrm{HWC}, \mathrm{HO}$ is some tuning parameter that allows us to probe the energies between that of the highest occupied state and the lowest unoccupied state. Picking $\lambda^{\alpha}=0$ corresponds to picking the Fermi level at the highest occupied state and $\lambda^{\alpha}=1$ corresponds to taking the Fermi level at the lowest unoccupied state. Another appealing choice is $\lambda^{\alpha}=1 / 2$, corresponding to taking the Fermi level right in the middle. For the time being, we will carry around the tuning parameters, but at some point we would like to pick specific values.

Note in particular that the ambiguity of the Fermi momentum disappears in the thermodynamic limit, because the difference between the highest occupied state and lowest unoccupied state becomes insignificant when $N \rightarrow \infty$. This is reflected in the fact that the ambiguity of the Fermi momentum is not discussed in Ref. [30], where also the concepts of the Fermi level and Fermi energy, which only equal each other in the thermodynamic limit, are used somewhat interchangeably. But if we want to derive energy expressions that apply to finite $N$, we should be careful when choosing the Fermi momentum.

\section{McGuire, Astrakharchik and Brouzos (PBC, thermodynamic limit)}

In Ref. 52] McGuire finds the following expression for the ground state energy of the interacting, free system 
subject to PBC:

$$
\Delta E_{g}^{\mathrm{f}}=\frac{\left(k_{F}^{\mathrm{PBC}}\right)^{2}}{2} \frac{\gamma}{\pi^{2}}\left[1-\frac{\gamma}{4}+\left(\frac{\gamma}{2 \pi}+\frac{2 \pi}{\gamma}\right) \tan ^{-1} \frac{\gamma}{2 \pi}\right],
$$

where $\gamma=g \pi / k_{F}^{\mathrm{PBC}}$. The result applies to all values of the interaction strength $g$, but in deriving Eq. (9) McGuire converts a sum to an integral letting $N \rightarrow \infty$ and $L \rightarrow \infty$ with the density $N / L$ held constant. This sum to integral conversion can be done in several ways introducing some degree of freedom in $k_{F}^{\mathrm{PBC}}$ consistent with the discussion in the previous section. Notice that picking $\lambda^{\mathrm{PBC}}=1 / 2$ would set $k_{F}^{\mathrm{PBC}}=(N-1) \pi / L$ for all $N$. This would imply a vanishing interaction correction for $N \rightarrow 1$ as it should. 61]

We now sketch how Astrakharchik and Brouzos implement LDA on McGuire's free energy expression (9) to find the energy of the trapped system [30]. 62 The LDA expression for the energy of the trapped system (7) yields

$$
\begin{aligned}
E_{g}^{\mathrm{t}} & \approx E_{0}^{\mathrm{t}}+\Delta E_{g}^{\mathrm{f}}\left(k_{F}^{\mathrm{HO}}\right) \\
& =\frac{(N-1)^{2}+1}{2} \\
& +\frac{\left(k_{F}^{\mathrm{HO}}\right)^{2}}{2} \frac{\gamma^{\mathrm{t}}}{\pi^{2}}\left[1-\frac{\gamma^{\mathrm{t}}}{4}+\left(\frac{\gamma^{\mathrm{t}}}{2 \pi}+\frac{2 \pi}{\gamma^{\mathrm{t}}}\right) \tan ^{-1} \frac{\gamma^{\mathrm{t}}}{2 \pi}\right],
\end{aligned}
$$

with $\gamma^{\mathrm{t}}=g \pi / k_{F}^{\mathrm{HO}}$. As before, we have to pick $\lambda^{\mathrm{HO}}=$ $1 / 2$ and thus $k_{F}^{\mathrm{HO}}=\sqrt{2(N-1)}$ in order to ensure that the energy correction vanishes for $N \rightarrow 1$. Since we are interested in the energy in the case of strong interaction, we expand the above general expression to first order in $1 / g$ :

$$
E_{g}^{\mathrm{t}} \approx \frac{N^{2}}{2}-\frac{1}{g} \mathcal{C}
$$

where the desired contact coefficient is given as

$$
\mathcal{C}=\frac{8 \sqrt{2}}{3 \pi}(N-1)^{3 / 2} .
$$

The above LDA expression is directly comparable to our exact results. Notice that the LDA method introduced ambiguities in the choice of the Fermi momenta, but that known physics could be used to restrict the choice and get an unambiguous final result. Unfortunately, this cannot be done in the calculations based on Oelkers' free results for a finite $N$ in $\mathrm{PBC}$ and $\mathrm{HWC}$.

\section{Oelkers (PBC and HWC, finite particle numbers)}

Before we compare the approximated expression in Eq. (12) with our exact calculations, we wish to derive two other approximated expression for the contact coefficient. These two expressions will rely on the results derived by Oelkers et al. in Ref. [53]. In this reference, the authors calculate the energy of a free strongly interacting system using PBC and HWC. Contrary to McGuire who assumed large $N$, these results are valid for finite (but even) $N$, which is what we ultimately are interested in. Our plan is now to apply the LDA method in order to get expressions for the trapped systems. We start by considering the case of PBC.

Periodic boundary condition. To first order in $1 / g$, we find the free ground state energy to be 63 .

$$
\begin{aligned}
E_{g}^{\mathrm{f}} & =\frac{\pi^{2}}{6 L^{2}}\left(N^{3}+2 N\right)\left[1-\frac{8}{g L}\right] \\
& =E_{\infty}^{\mathrm{f}}-\frac{1}{g} \frac{4 \pi^{2}}{3 L^{3}}\left(N^{3}+2 N\right) .
\end{aligned}
$$

We now follow the mapping prescription (8) and map $E_{\infty}^{\mathrm{f}} \mapsto E_{\infty}^{\mathrm{t}}=N^{2} / 2$ and $k_{F}^{\mathrm{PBC}} \mapsto k_{F}^{\mathrm{HO}}$. Using the expressions for $k_{F}^{\mathrm{PBC}}$ and $k_{F}^{\mathrm{HO}}$, we can rewrite the mapping as

$$
\frac{1}{L} \mapsto \frac{\sqrt{2\left(N-\frac{3}{2}+\lambda^{\mathrm{HO}}\right)}}{\pi\left(N-2+2 \lambda^{\mathrm{PBC}}\right)},
$$

where we have kept the tuning parameters unspecified. Applying the above substitution to the result in (13), we obtain the energy of the trapped system within LDA:

$$
E_{g}^{\mathrm{t}} \approx \frac{N^{2}}{2}-\frac{1}{g} \frac{8 \sqrt{2}}{3 \pi}\left(N^{3}+2 N\right) \frac{\left(N-\frac{3}{2}+\lambda^{\mathrm{HO}}\right)^{3 / 2}}{\left(N-2+2 \lambda^{\mathrm{PBC}}\right)^{3}} .
$$

In the thermodynamic limit, $N \rightarrow \infty$, the contact coefficient read off Eq. (15) and the one in Eq. (12) tend towards the same asymptote given by $\mathcal{C}_{\infty}=8 \sqrt{2} /(3 \pi) N^{3 / 2}$, regardless of the choice of the tuning parameters. This is indeed reassuring, but we are mostly interested in results for small $N$. Eq. (15) is derived for even $N$, but let us extend the domain also to odd $N$ in order to restrict the tuning parameters by requiring the vanishing of the contact coefficient for $N=1$. Since we also require $\mathcal{C}$ to be non-divergent for finite $N$, we end up with $\lambda^{\mathrm{HO}}=1 / 2$ and $\left.\left.\lambda^{\mathrm{PBC}} \in\right] 0 ; 1 / 2[\cup] 1 / 2 ; 1\right]$. While this fixes $\lambda^{\mathrm{HO}}$, there is still ambiguity in $\lambda^{\mathrm{PBC}}$. Choosing $\lambda^{\mathrm{PBC}}=1$ yields

$$
\mathcal{C}=\frac{8 \sqrt{2}}{3 \pi}\left(N^{3}+2 N\right) \frac{(N-1)^{3 / 2}}{N^{3}} .
$$

Hard wall condition. The ground state energy with HWC is found in Ref. [53] 64]:

$$
E_{g}^{\mathrm{f}}=\frac{\pi^{2}}{12 L^{2}}\left(2 N^{3}-3 N^{2}+N\right)\left[1-\frac{8}{g L} \cos ^{2}\left(\frac{\pi}{2 N}\right)\right] .
$$

This result is consistent with the energy found in Ref. 54] in the thermodynamic limit $(N, L \rightarrow \infty$ and $N / L$ constant). In a completely similar way as before, mapping $E_{\infty}^{\mathrm{f}} \mapsto E_{\infty}^{\mathrm{t}}=N^{2} / 2$ and $k_{F}^{\mathrm{HWC}} \mapsto k_{F}^{\mathrm{HO}}$ yields the energy 


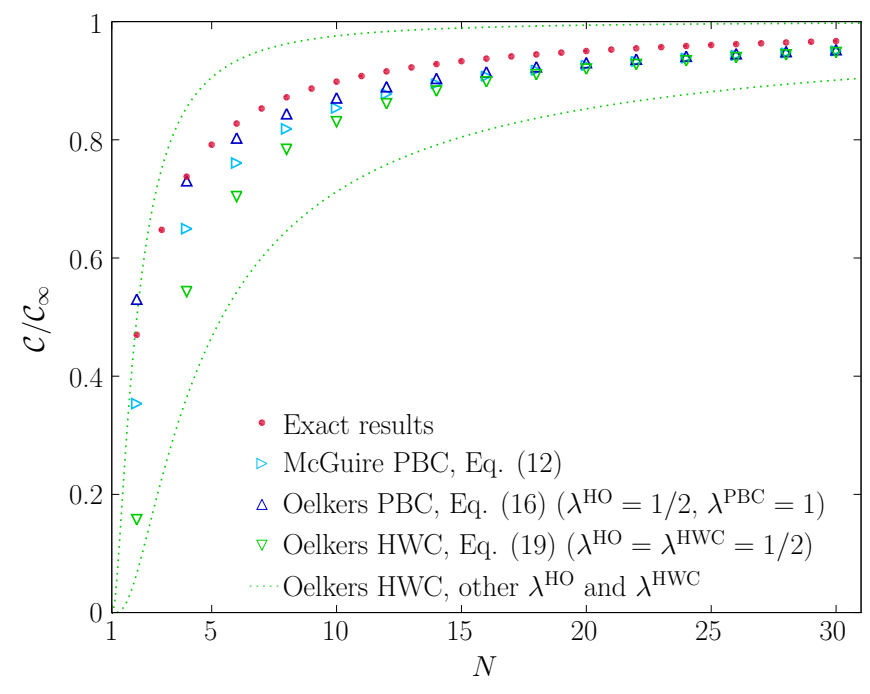

FIG. 3: Comparing the approximated contact coefficients based on the results of Oelkers et al. 53] and McGuire 52] with our exact results, scaled with the common asymptote for $N \rightarrow \infty$. For Oelkers HWC we also change the tuning parameters to see how that influences the result. The dotted lines show the choices $\lambda^{\mathrm{HO}}=1$ and $\lambda^{\mathrm{HWC}}=1 / 4$ (upper curve) and $\lambda^{\mathrm{HO}}=1 / 2$ and $\lambda^{\mathrm{HWC}}=1$ (lower curve), where we for clarity have taken $N$ to be a continuous parameter.

of the trapped system:

$$
\begin{aligned}
E^{\mathrm{t}}= & \frac{N^{2}}{2}-\frac{1}{g} \frac{8 \sqrt{2}}{3 \pi}\left(N^{3}-\frac{3}{2} N^{2}+\frac{1}{2} N\right) \\
& \times \frac{\left(N-\frac{3}{2}+\lambda^{\mathrm{HO}}\right)^{3 / 2}}{\left(N-1+\lambda^{\mathrm{HWC}}\right)^{3}} \cos ^{2}\left(\frac{\pi}{2 N}\right) .
\end{aligned}
$$

As expected, the contact coefficient goes towards the common asymptote $\mathcal{C}_{\infty}$ in the limit $N \rightarrow \infty$. Again we wish to extend the result to odd $N$, not just even $N$. To ensure that the contact coefficient is real and well-defined for any integer $N \geq 1$ and zero at $N=1$, we should pick either $\lambda^{\mathrm{HO}}=1 / 2$ and $\lambda^{\mathrm{HWC}}=0$ or $\lambda^{\mathrm{HO}} \in[1 / 2 ; 1]$ and $\left.\left.\lambda^{\mathrm{HWC}} \in\right] 0 ; 1\right]$. For the choices $\lambda^{\mathrm{HO}}=\lambda^{\mathrm{HWC}}=1 / 2$, we get

$$
\mathcal{C}=\frac{8 \sqrt{2}}{3 \pi}\left(N^{3}-\frac{3}{2} N^{2}+\frac{1}{2} N\right) \frac{(N-1)^{3 / 2}}{\left(N-\frac{1}{2}\right)^{3}} \cos ^{2}\left(\frac{\pi}{2 N}\right) .
$$

In deriving Eqs. (16) and (19), we had to specify values for the tuning parameters, but notice that we could just as well have chosen other values within certain bounds.

\section{E. Comparing contact coefficients}

We now wish to compare the contact coefficients that we derived in the previous two subsections. First of all, we want to see, whether the approximated results compare well with our exact results. Secondly, we want to investigate how the choice of the tuning parameters interferes with the result for small values of $N$. On Figure 3 we have shown our exact results compared with the three LDA expressions in Eqs. (12), (16) and (19).

We see that the LDA results tend to undershoot compared to our exact results. We also wanted to compare HWC results and PBC results, because our intuition told us that a hard wall box 'looked more' like a harmonic trap than a free periodic potential did. Contrary to our expectation, the HWC result does not appear to be better than the PBC results. On the other hand, the ambiguity in the LDA results introduced by the choice of the Fermi level and manifested in the tuning parameters makes it impossible to compare $\mathrm{HWC}$ and $\mathrm{PBC}$ in a unique way: We have to choose values for the tuning parameters. On Figure 3 we also show the HWC result for other values of the tuning parameters, resulting in drastically altered contact coefficients. Even for $N \sim 30$, the choice of the tuning parameters matters, but eventually it will become insignificant as $N$ becomes larger and larger.

In our opinion this constitute the most important lesson about the LDA method: For values of $N$ that realistically can be probed experimentally today or in the coming years, the partially arbitrary position of the Fermi level has a very large effect on the LDA predicted contact coefficient. In the light of this conclusion, it seems like an improbable stroke of luck that McGuire's result found in the limit $N \rightarrow \infty$ works so well when extrapolated to the finite $N$ regime.

\section{COMPARING MODELS TO EXPERIMENT}

The ground state energy for the impurity system was found experimentally in Ref. [20] for $N=2, \ldots, 6$ and $g=0.36,1.14$ and 2.80. Naturally, we want to examine how the theories that assume large $N$ and/or $g$ compare with each other and the experimental results.

McGuire assumes that $N \rightarrow \infty$, so we will use Eqs. (10) and (11) as the approximated theoretical energies assuming that $g$ is finite and very large, respectively. We use our exact contact coefficients as a theoretical prediction assuming finite $N$, but with $g$ being very large. We also have exact numerical calculations of the energy for finite $N$ and finite $g$, but this is a very demanding computation, so we only have reliable results for $N \leq 11$ (using the effective interaction method recently introduced to address Fermi [35] and Bose systems [55]). These four theoretical predictions exhaust the four combinations of finite/infinite $g$ and $N$. We compare them with the experimental data on on Figure 4 .

Not surprisingly, the most general theory obtained from full numerical calculations for finite values of $g$ and $N$ is in very good agreement with the experimental data. But we also see that the theory assuming finite $g$ and $N \rightarrow \infty$ works equally well for very small values of $N$. 

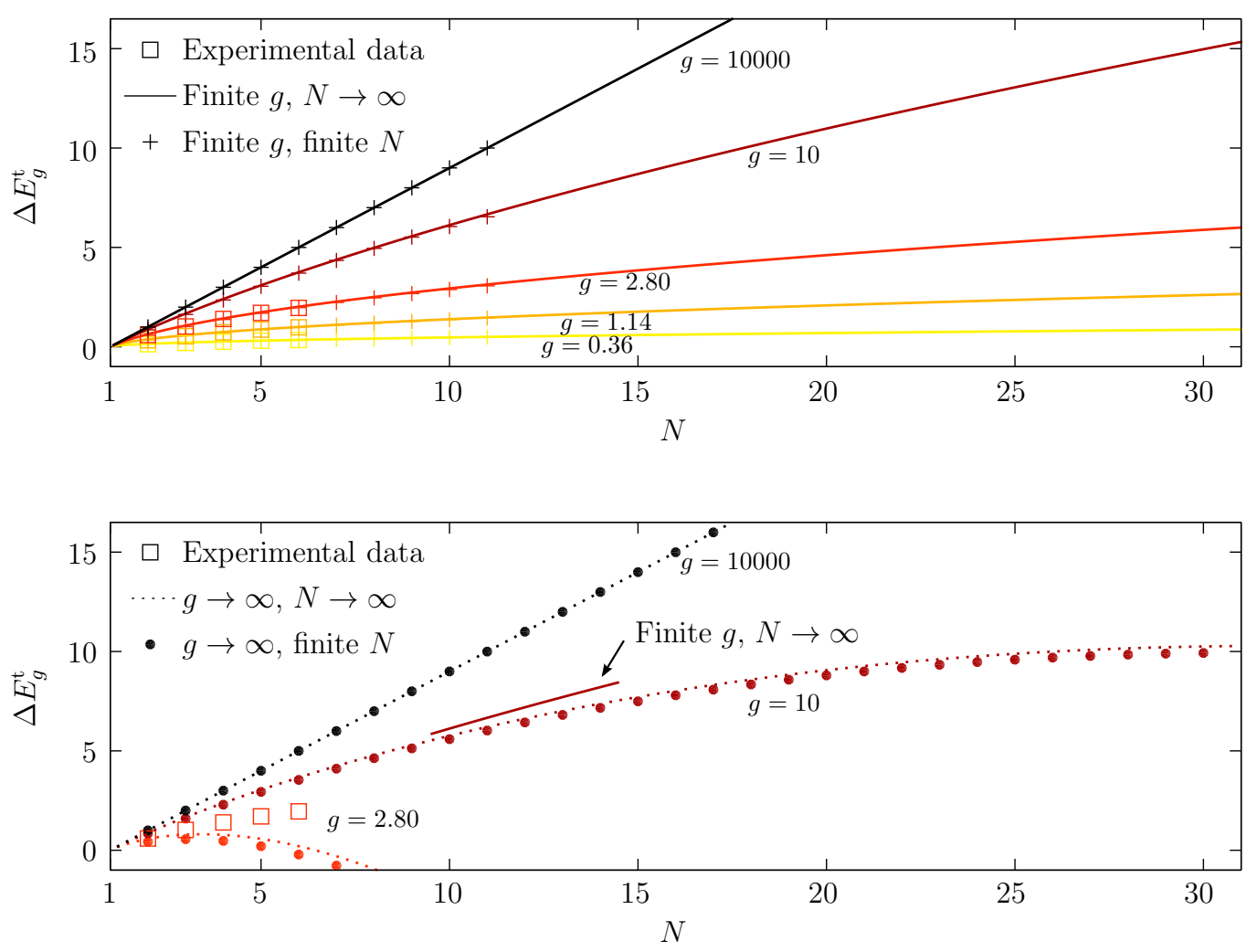

FIG. 4: Interaction correction to the energy of the non-interacting system as function of the total number of particles. The colors refer to different values of the interaction strength. On the top figure, we compare experimental data with theories for finite $g$. For $N \leq 11$ we have reliable results from a full numerical treatment for finite $g$ and finite $N(+)$. Notice that the agreement with the McGuire formula for finite $g$ and $N \rightarrow \infty$ in Eq. (10) (-) is excellent in the few-body regime and is expected to improve for increasing $N$. On the bottom figure, we compare experimental data with theories assuming $g \rightarrow \infty$. We compare the results for finite $N$ using Eq. (4) and the exact geometric coefficients in Appendix $\mathrm{A}(\bullet)$ and the McGuire formula in the limit $N \rightarrow \infty$ in Eq. (11) ( ...). To illustrate how the $g \rightarrow \infty$ theories fall off for increasing $N$ we also show a piece of the finite $g$ McGuire formula for $g=10$ on the bottom plot.

This is indeed an unexpected result, especially when we recall from the previous section that the exact position of the Fermi level chosen in the LDA could have a huge impact on the final result for small $N$.

Let us now examine the theories assuming $g \rightarrow \infty$. We immediately see that our exact result and the LDA result are in very good agreement as a direct consequence of the agreement between the exact contact coefficients and the McGuire result in Figure 3 We know that the interaction correction goes like $\Delta E_{g}^{\mathrm{t}} \sim-N^{3 / 2} / g$ for large $N$, so for suitable large $N$ and/or small $g$ the theory must break down (at some point the interaction correction even becomes negative). This is clearly seen when comparing the top and bottom part of Figure 4. It is seen that strongly interacting theories deviate from the general theories when $N$ increases or $g$ decreases. The interesting question is then for which $N$ and $g$ does the strongly interaction theory describe nature? The largest experimental interaction strength probed in Ref. [20] is $g=2.80$, but as is seen on the bottom part of Figure 4 theory and experiment only matches for $N=2$, so this is clearly not very strong interaction. For $g=10$, we get a good agreement between the theories assuming finite $g$ and large $g$ for $N \lesssim 12$, but then the theories split up. We find it surprising how quickly the strong interaction theory breaks down.

\section{SUMMARY}

We have considered a trapped one-dimensional Fermi system interacting with a single impurity of the same mass from the point of view of energetics. We have discussed how the local density approximation served as a way to map the ground state energy of the free system into the ground state energy of the trapped system. We 
also saw that the method could introduce ambiguous results for finite $N$ that heavily influenced the predicted energies. This naturally lead to a skepticism for LDA results for small $N$, which are currently used to describe experiments [20]. Despite the erratic nature of the LDA results, the energy expression based on McGuire's result works surprisingly well[20, 30, 52].

We also discussed how the strong interaction limit must be approached with caution as the particle number increase. Comparing theories for finite interaction and strong interaction, we found that $g=2.80$ cannot be classified as strong interaction, and for $g=10$ the theories start deviating significantly already around $N \sim 12$. This is consistent with the observation that the natural interaction strength in the harmonic trap is proportional to $g / \sqrt{N}$ [30] (see also the earlier discussion in Ref. [57]).

Remark. During preparation of the revised version of this manuscript, we became aware of a paper by Deuretzbacher et al. [58] which describes a method for computing the geometric coefficients and presents results for harmonic traps with up to 30 particles using a method that has some common features with the computational approach used here.

\section{Acknowledgments}

The authors wish to thank A. S. Dehkharghani and G. Zürn for supplying us with numerical and experimental data, respectively. We would also like to thank A. G.
Volosniev for discussions and for reading and commenting on the manuscript. This work is supported in part by the Danish Council for Independent Research DFF Natural Sciences and the DFF Sapere Aude program, as well as by a grant from the Carlsberg Foundation.

\section{Appendix A: Geometric coefficients for a harmonic trap}

Here we tabulate the geometric coefficients, $\alpha_{i}$, in Eq. (2) in harmonic oscillator units. For completeness we note that the explicit unit is $(\hbar \omega)^{2} b$, where $b=\sqrt{\hbar / m \omega}$ is the standard oscillator length. To the right of every indicated value of $N$ we record $\alpha_{1}, \alpha_{2}, \ldots$ up to $\alpha_{N / 2}$ for $N$ even and $\alpha_{(N-1) / 2}$ for $N$ odd, continuing on the next line if necessary. The remaining geometric coefficients are given by the symmetry property $\alpha_{k}=\alpha_{N-k}$ for $k=1, \ldots, N-1$ as there are $N-1$ coefficients for each $N$. We have calculated all $N-1$ coefficients for each $N$ and used the symmetry property to estimate the numerical precision on the coefficients by noting the digits in $\alpha_{k}$ in agreement with the digits in $\alpha_{N-k}$. In this appendix we have at most given 10 significant figures even though many of our calculations are even more precise. Generally a higher precision can be achieved for the price of higher calculation time. The coefficients given here agree with previous calculations which have been done for 10 particles or less [36, 37, 47]. 


\begin{tabular}{|c|c|c|c|c|c|c|c|c|}
\hline$N$ & $\alpha_{1}=\alpha_{N-1}$ & $\alpha_{2}=\alpha_{N-2}$ & & & & & & \\
\hline 2 & $\sqrt{2 / \pi}=$ & & & & & & & \\
\hline & 0.7978845608 & & & & & & & \\
\hline 3 & $3^{3} /\left(2^{\frac{7}{2}} \sqrt{\pi}\right)=$ & & & & & & & \\
\hline & 1.346430196 & & & & & & & \\
\hline 4 & 1.787645708 & 2.346508058 & & & & & & \\
\hline 5 & 2.166057718 & 3.177197531 & & & & & & \\
\hline 6 & 2.5021784 & 3.902098540 & 4.357116131 & & & & & \\
\hline 7 & 2.807397825 & 4.552904442 & 5.400326410 & & & & & \\
\hline 8 & 3.088795182 & 5.148133894 & 6.345017625 & 6.738585693 & & & & \\
\hline 9 & 3.351118130 & 5.69971481 & 7.214307411 & 7.959044430 & & & & \\
\hline 10 & 3.597730090 & 6.215859197 & 8.023484036 & 9.08789432 & 9.439679835 & & & \\
\hline 11 & 3.831114914 & 6.702502948 & 8.78328981 & 10.14280881 & 10.81522991 & & & \\
\hline 12 & 4.053167612 & 7.164099681 & 9.501616401 & 11.13640807 & 12.10509843 & 12.42610595 & & \\
\hline 13 & 4.265372369 & 7.604090937 & 10.18446319 & 12.078086 & 13.32332240 & 13.94120413 & & \\
\hline 14 & 4.468916965 & 8.025200651 & 10.83651694 & 12.97506385 & 14.48046497 & 15.37564625 & 15.67276485 & \\
\hline 15 & 4.664769312 & 8.429628019 & 11.46152119 & 13.83303366 & 15.584720 & 16.74091572 & 17.31574325 & \\
\hline 16 & 4.853730361 & 8.819178529 & 12.06252191 & 14.65657900 & 16.64259962 & 18.04599032 & 18.88235477 & 19.16023435 \\
\hline 17 & 5.036471720 & 9.195355790 & 12.64203656 & 15.44945457 & 17.65938206 & 19.2980535 & 20.38221388 & 20.92192258 \\
\hline 18 & 5.213563048 & 9.559427571 & 13.20217385 & 16.21478205 & 18.63941758 & 20.50296313 & 21.82305903 & 22.61094361 \\
\hline & 22.87289739 & & & & & & & \\
\hline 19 & 5.385492433 & 9.912474388 & 13.74472084 & 16.95518993 & 19.58634200 & 21.66557213 & 23.211233 & 24.23549760 \\
\hline & 24.74584444 & & & & & & & \\
\hline 20 & 5.552681814 & 10.25542595 & 14.27120748 & 17.67291605 & 20.50323106 & 22.78995562 & 24.55201753 & 25.80233751 \\
\hline & 26.54935034 & 26.79783773 & & & & & & \\
\hline 21 & 5.715498860 & 10.58908900 & 14.78295544 & 18.36988415 & 21.39271425 & 23.87957538 & 25.84986282 & 27.31710 \\
\hline & 28.29051532 & 28.77583530 & & & & & & \\
\hline 22 & 5.874266240 & 10.91416887 & 15.28111566 & 19.04776222 & 22.25706048 & 24.93740208 & 27.10856575 & 28.7845843 \\
\hline & 29.97529569 & 30.68723324 & 30.92413925 & & & & & \\
\hline 23 & 6.029268959 & 11.23128639 & 15.76669765 & 19.70800760 & 23.09824363 & 25.96600770 & 28.33139508 & 30.20885539 \\
\hline & 31.6087 & 32.53825677 & 33.00191145 & & & & & \\
\hline 24 & 6.180760221 & 11.54099143 & 16.24059255 & 20.35190233 & 23.91799356 & 26.96763670 & 29.52118965 & 31.59345070 \\
\hline & 33.19521851 & 34.33420853 & 35.01560955 & 35.24241668 & & & & \\
\hline 25 & 6.328966176 & 11.84377367 & 16.70359169 & 20.98058126 & 24.71783628 & 27.94426160 & 30.68043376 & 32.94144468 \\
\hline & 34.73846196 & 36.07965 & 36.97074938 & 37.41540822 & & & & \\
\hline 26 & 6.474089790 & 12.14007146 & 17.15640150 & 21.59505463 & 25.49912605 & 28.89762693 & 31.81131634 & 34.25553479 \\
\hline & 36.24176315 & 37.778550 & 38.87208994 & 39.5265884 & 39.7444875 & & & \\
\hline 27 & 6.616314036 & 12.43027900 & 17.59965570 & 22.19622631 & 26.26307117 & 29.82928451 & 32.9157779 & 35.5381033 \\
\hline & 37.70800940 & 39.43437 & 40.72377 & 41.58088866 & 42.00871 & & & \\
\hline 28 & 6.755804540 & 12.71475230 & 18.03392534 & 22.78490885 & 27.01075509 & 30.7406219 & 33.995548 & 36.7912671 \\
\hline & 39.139756 & 41.05017 & 42.5294331 & 43.58261037 & 44.21317022 & 44.42314030 & & \\
\hline 29 & 6.892711795 & 12.99381416 & 18.45972710 & 23.3618358 & 27.7431536 & 31.63288 & 35.05217 & 38.016916 \\
\hline & 40.53927926 & 42.6286 & 44.29227783 & 45.5355 & 46.36230034 & 46.775 & & \\
\hline 3 & 7.027173023 & 13.26775837 & 18.8775302 & 23.9276722 & 28.461149 & 32.50720 & 36.0870 & 39.2167 \\
\hline & 41.90861428 & 44.172 & 46.01515 & 47.44300481 & 48.46001639 & 49.069 & 49.271 & \\
\hline
\end{tabular}


[1] I. Bloch, J. Dalibard, and W. Zwerger, Rev. Mod. Phys. 80, 885 (2008).

[2] M. Lewenstein et al., Adv. Phys. 56, 243 (2007).

[3] T. Esslinger, Ann. Rev. Cond. Mat. Phys. 1, 129 (2010).

[4] M. A. Baranov, M. Dalmonte, G. Pupillo, and P. Zoller, Chem. Rev. 112, 5012 (2012).

[5] N. T. Zinner and A. S. Jensen, J. Phys. G:Nucl. Part. Phys. 40, 053101 (2013).

[6] M. Olshanii, Phys. Rev. Lett. 81, 938 (1998).

[7] H. Moritz, T. Stöferle, M. Köhl, and T. Esslinger, Phys. Rev. Lett. 91, 250402 (2003).

[8] T. Stöferle, H. Moritz, C. Schori, M. Köhl, and T. Esslinger, Phys. Rev. Lett. 92, 130403 (2004).

[9] T. Kinoshita, T. Wenger, and D. S. Weiss, Science 305, 1125 (2004).

[10] B. Paredes et al., Nature 429, 277 (2004).

[11] T. Kinoshita, T. Wenger, and D. S. Weiss, Nature 440 , 900 (2005).

[12] E. Haller et al., Science 325, 1224 (2009).

[13] E. Haller et al., Nature 466, 597 (2010).

[14] G. Pagano et al., Nature Phys. 10, 198 (2014).

[15] L. W. Tonks, Phys. Rev. 50, 955 (1936).

[16] M. D. Girardeau, J. Math. Phys. 1, 516 (1960).

[17] F. Serwane et al., Science 332, 336 (2011).

[18] G. Zürn et al., Phys. Rev. Lett. 108, 075303 (2012).

[19] G. Zürn et al., Phys. Rev. Lett. 111, 175302 (2013).

[20] A. Wenz et al., Science 342, 457 (2013).

[21] S. Murmann et al., Phys. Rev. Lett. 114, 080402 (2015).

[22] S. Murmann et al.,Phys. Rev. Lett. 115, 215301 (2015).

[23] L. Guan, S. Chen, Y. Wang, and Z.-Q. Ma, Phys. Rev. Lett. 102, 160402 (2009).

[24] C. N. Yang, Chin. Phys. Lett. 26, 120504 (2009).

[25] M. D. Girardeau, Phys. Rev. A 82, 011607(R) (2010).

[26] L. Guan and S. Chen, Phys. Rev. Lett. 105, 175301 (2010).

[27] D. Rubeni, A. Förster, and I. Roditi, Phys. Rev. A 86, 043619 (2012).

[28] I. Brouzos and P. Schmelcher, Phys. Rev. A 87, 023605 (2013).

[29] P. O. Bugnion and G. J. Conduit, Phys. Rev. A 87, 060502(R) (2013).

[30] G. E. Astrakharchik and I. Brouzos, Phys. Rev. A 88, 021602(R) (2013).

[31] S. E. Gharashi and D. Blume, Phys. Rev. Lett. 111, 045302 (2013).

[32] T. Sowiński, T. Graß, O. Dutta, and M. Lewenstein, Phys. Rev. A 88, 033607 (2013).

[33] S. E. Gharashi, X. Y. Yin, and D. Blume, Phys. Rev. A 89, 023603 (2014).

[34] A. G. Volosniev et al., Few-Body Syst. 55, 839 (2014).

[35] E. J. Lindgren et al., New J. Phys. 16, 063003 (2014).

[36] A. G. Volosniev et al., Nature Commun. 5, 5300 (2014).

[37] F. Deuretzbacher et al., Phys. Rev. A 90, 013611 (2014).

[38] X. Cui and T.-L. Ho, Phys. Rev. A 89, 023611 (2014).
[39] E. V. H. Doggen, A. Korolyuk, P. Törmä, and J. J. Kinnunen, Phys. Rev. A 89, 053621 (2014).

[40] N. J. S. Loft et al., Eur. Phys. J. D 69, 65 (2015).

[41] F. Nur Ünal, B. Hetényi, and M. Ö. Oktel, Phys. Rev. A 91, 053625 (2015).

[42] R. Lundmark, C. Forssén, and J. Rotureau, Phys. Rev. A 91, 041601(R) (2015).

[43] S. E. Gharashi, X. Y. Yin, and D. Blume, Phys. Rev. A 91, 013620 (2015).

[44] A. G. Volosniev et al., Phys. Rev. A 91, 023620 (2015).

[45] L. Yang, L. Guan, and H. Pu, Phys. Rev. A 91, 043634 (2015).

[46] T. Sowiński, M. Gajda, and K. Rzążewski, Europhys. Lett. 109, 26005 (2015).

[47] J. Levinsen, P. Massignan, G. M. Bruun, and M. M. Parish, Science Adv. 1, e1500197 (2015).

[48] A. G. Volosniev, H.-W. Hammer, and N. T. Zinner, Phys. Rev. B 93, 094414 (2016).

[49] T. Grining et al., Phys. Rev. A 92, 061601(R) (2015).

[50] S. Tan, Ann. Phys. 323, 2952 (2008); 323, 2971 (2008); 323, 2987 (2008).

[51] M. Valiente, N. T. Zinner, and K. Mølmer, Phys. Rev. A 86, 043616 (2012).

[52] J. B. McGuire, J. Math. Phys. 6, 432 (1965).

[53] N. Oelkers, M. T. Batchelor, M. Bortz and X.-W. Guan, J. Phys. A: Math. Gen. 39, 1073 (2006).

[54] X.-W. Guan and Z.-Q. Ma, Phys. Rev. A 85, 033632 (2012).

[55] A. S. Dehkharghani et al., Scientific Reports 5, 10675 (2015).

[56] N. J. S. Loft et al., arXiv:1603.02662 (2016)

[57] G. E. Astrakharchik, D. Blume, S. Giorgini, and L. P. Pitaevskii, Phys. Rev. Lett. 93, 050402 (2004).

[58] F. Deuretzbacher, D. Becker, and L. Santos, arXiv:1602.06816 (2016).

[59] The program and source code can be downloaded here

[60] The impurity is occupying the state of lowest energy, but this is irrelevant for the determination of the Fermi level.

[61] We have to consider the limit of Eq. (9) to avoid zero division at $k_{F}^{\mathrm{PBC}}=0$.

[62] Notice that in Ref. [30] the number of majority particles is denoted by $N$, and not $N-1$ as in our convention.

[63] We note a sign error in Eq. (14) of Ref. [53] as the denominator should read $\gamma_{i}-\gamma_{j}-i$. Also there appear to be a typo in Eq. (16) as the first factor being the energy of $N$ Fermi particles, $E_{\infty}^{\mathrm{f}}$, is recorded to be $\pi^{2} /\left(6 L^{2}\right)\left(N^{3}-N\right)$. But this expression is only valid for $N$ odd, and since $N$ even is assumed, the first factor should be $\pi^{2} /\left(6 L^{2}\right)\left(N^{3}+2 N\right)$. Finally note the difference of a factor of $1 / 2$ in their definition of the energy.

[64] We note a sign error on the second term in the front factor in Eq. (19) in Ref. [53]. 